\title{
Asymmetricity of ground-based GPS slant delay data
}

\author{
R. Eresmaa, H. Järvinen, S. Niemelä, and K. Salonen \\ Finnish Meteorological Institute, Erik Palménin aukio 1, 00100 Helsinki, Finland \\ Received: 19 December 2006 - Published in Atmos. Chem. Phys. Discuss.: 27 February 2007 \\ Revised: 30 May 2007 - Accepted: 8 June 2007 - Published: 20 June 2007
}

\begin{abstract}
The ground-based measurements of the Global Positioning System (GPS) allow estimation of the tropospheric delay along the slanted signal paths through the atmosphere. The meteorological exploitation of such slant delay (SD) observations relies on the hypothesis of azimuthal asymmetry of the information content. This article addresses the validity of the hypothesis.

A new concept of asymmetricity is introduced for studying the SD observations and their model counterparts. The asymmetricity is defined as the ratio of the absolute asymmetric delay component to total SD. The model counterparts are determined from 3-h forecasts of a numerical weather prediction (NWP) model, run with four different horizontal resolutions. The SD observations are compared with their model counterparts with emphasis on cases of high asymmetricity in order to see whether the observed asymmetry is a real atmospheric signature.

The asymmetricity is found to be of the order of a few parts per thousand. Thus, the asymmetric delay component barely exceeds the assumed standard deviation of the SD observation error. However, the observed asymmetric delay components show a statistically significant meteorological signal. Benefit of the asymmetric SD observations is therefore expected to be taken in future, when NWP systems will explicitly represent the small-scale atmospheric features revealed by the SD observations.
\end{abstract}

\section{Introduction}

Dense networks of ground-based receivers of the Global Positioning System (GPS) constitute a meteorological observing system for numerical weather prediction (NWP) (e.g. Elgered et al., 2005). The applications of ground-based GPS

Correspondence to: R. Eresmaa

(reima.eresmaa@fmi.fi) meteorology show potential especially on short-range NWP, where the forecast lead time is of the order of 3-18 h. This time scale corresponds to atmospheric phenomena in horizontal length scales of a few hundreds of kilometers or less. Geodetic processing of raw GPS measurements results in delay observations. These are measures of atmospheric refractivity integrated over either a vertical column (zenith total delay, ZTD observations; e.g. Bevis et al., 1992) or a slanted signal path between a satellite and a receiver station (slant delay, SD observations; e.g. de Haan et al., 2002). Several studies show that data assimilation of the ZTD observations, processed in near-real-time, can result in a positive NWP forecast impact on humidity and precipitation in synoptic scales (e.g. De Pondeca and Zou, 2001; Vedel and Huang, 2004; Elgered et al., 2005). Considerably fewer reports are available on data assimilation of the SD observations. As the existing dense GPS receiver station networks do not yet cover areas large enough for NWP, these studies are mainly conducted by using hypothetical observations (MacDonald et al., 2002; Ha et al., 2003; Liu and Xue, 2006).

The ZTD observations exhibit no information on azimuthal asymmetry of atmospheric refractivity field. In presence of humidity gradients, data assimilation of the ZTD observations in a high resolution NWP system can therefore be considered suboptimal. In theory, the SD observations are capable of detecting the azimuthal asymmetry. Since strong humidity gradients are typical fingerprints of severe weather, exploitation of the SD observations is an attractive development. Forecasting of severe weather is considered as one of the main challenges for the current NWP activities (Hollingsworth et al., 2002; Bouttier, 2004).

An SD observation can be thought to consist of symmetric and asymmetric components (de Haan et al., 2002). It is obvious that the asymmetric component represents atmospheric phenomena in considerably finer scales than the symmetric component. Consequently, the SD observations are expected to show potential on NWP in very high spatial resolution,

Published by Copernicus Publications on behalf of the European Geosciences Union. 
but not necessarily in the synoptic scales. So far, there has been only little evidence that the currently operational NWP systems can benefit from the asymmetric property of the SD observations.

This article assesses the potential of the asymmetric delay components from data assimilation point of view. Answers are searched especially to the following questions. First, how large is the contribution of the azimuthally asymmetric information to the SD observations? Second, is the azimuthal asymmetry in the SD observations related to real atmospheric asymmetry? Third, are the currently operational NWP systems, with horizontal grid spacings of around $10-20 \mathrm{~km}$, able to represent the scales appropriate for extracting the asymmetric information? Fourth, can the NWP models' representation of the azimuthal asymmetry be improved by increasing the horizontal resolution?

The structure of this article is as follows: The used SD observations and the NWP model are described in Sect. 2. Following Sects. 3 and 4 focus on statistical properties of the asymmetric components of the SD observations (Sect. 3) and NWP model counterparts to the SD observations (Sect. 4). In Sect. 5, extreme cases of the azimuthal asymmetry in the observations and in the model counterparts are intercompared. Section 6 presents the conclusions.

\section{Methodology and used data}

\subsection{SD observations and their NWP model counterparts}

The SD observations used in this study are processed at the Technical University of Delft, the Netherlands. The observations originate from 17 receiver stations in the North-Western Europe over the time period 1-24 May 2003. Due to computational limitations of the NWP model, a subset of 296604 observations from 13 receiver stations, with a time interval of $10 \mathrm{~min}$, is used. The receiver station locations are shown in Fig. 1.

The fundamental assumption behind the SD processing is that the fitting residuals of the geodetic network solution are indicative of the atmospheric asymmetry (de Haan et al., 2002). This assumption allows the usage of a two-step procedure for processing. First, the symmetric component of SD is estimated as part of the network solution, using the leastsquares fitting. Second, the fitting residuals are added on top of the symmetric component to obtain the final SD. Formally one can write

$$
\mathrm{SD}=\underbrace{m_{h} \mathrm{ZHD}+m_{w} \mathrm{ZWD}}_{\text {Symmetric component }}+\delta_{r}^{s},
$$

where $m_{h}$ and $m_{w}$ are the hydrostatic and wet mapping functions, respectively, ZHD and ZWD are the zenith hydrostatic and wet delays, and $\delta_{r}^{s}$ is the fitting residual, interpreted as the asymmetric component of SD, corresponding to the satel- lite $s$ and the receiver $r$. The processing applies the mapping functions proposed by Niell (1996).

The applied methodology for the processing has been criticised in geodetic literature. In particular, Elosegui and Davis (2004) showed by a simulation study, that the azimuthally asymmetric delay component cannot be completely retrieved from the fitting residuals of the network solution. Moreover, a gross measurement error in a single raw observation has a considerable impact on the other SD observations processed for the same receiver at the same time. This problem relates to general properties of any least squares fit, and it degrades the potential of the SD observations for any application, not only for NWP.

The model counterparts to the SD observations are produced from the output of the High Resolution Limited Area Model (Hirlam; Undén et al., 2002). Three hour Hirlam forecasts are transformed from the model grid to the observation space by applying the non-linear SD observation operator (Eresmaa and Järvinen, 2006). The hydrostatic forecast model has been run with four different model resolutions. The initial state for the model integration is obtained by the three-dimensional variational data assimilation (3D-Var) system of HiRlam (Gustafsson et al., 2001; Lindskog et al., 2001) separately for each model run. The SD observations have not been assimilated in the model. The first model run applies 40 model levels in vertical, horizontal grid spacing of $22 \mathrm{~km}$ and the operational analyses of the European Centre for Medium-range Weather Forecasts (ECMWF) as the lateral boundary condition. For the subsequent nested model runs, the grid spacing is halved in horizontal, and the lateral boundary conditions are retrieved from the NWP analyses obtained from the previous model run with a coarser grid. Consequently, the horizontal grid spacings in the nested runs are $11,5.6$ and $2.8 \mathrm{~km}$, respectively.

Use of a non-hydrostatic NWP system, rather than the hydrostatic HIRLAM system, would in theory be more justified for horizontal grid spacings below $5 \mathrm{~km}$ (Niemelä and Fortelius, 2005). There is also a non-hydrostatic version of the HIRLAm model, but it is not applied here. This is motivated as follows: since the primary objective of running the model in a nested mode is to gain insight specifically into the role of the horizontal resolution in the SD modelling, the details of the four model runs are kept as close as possible to each other. Different model dynamics and physical parameterizations would be likely to confuse the interpretation of the results. Furthermore, since the area of interest is relatively flat and the studied period is not characterized by strong convective activity, the non-hydrostatic effects are believed to be weak. Role of the non-hydrostatic modelling is considered as a separate research issue, and it is not addressed in this study. 


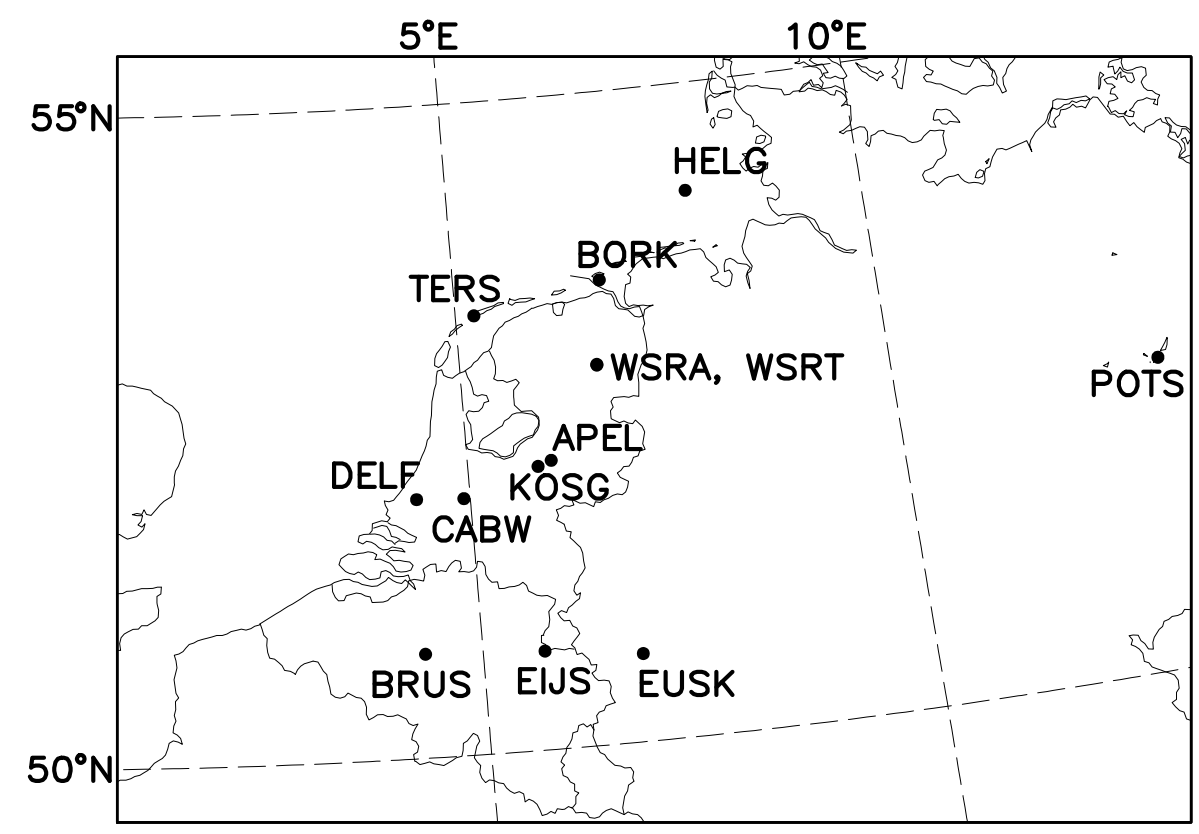

Fig. 1. The HIRLAM NWP model domain used in the run with grid spacing of $2.8 \mathrm{~km}$. The GPS receiver station locations are marked with dots.

\subsection{Determination of the asymmetric SD components}

Both the symmetric SD components and the fitting residuals are available in the present SD observation data set. Therefore, the asymmetric SD components would be readily available for examination. In contrast, since the SD observation operator involves no least squares fitting, the NWP model counterpart data sets contain only the total SD values. For the sake of comparability, the asymmetric delay components from the observations and from the model counterparts are extracted in a similar manner using the following procedure. Note that the term "observable" is used here to refer to both the SD observations and the NWP model counterparts at the four horizontal grid resolutions.

1. The SD observables are classified in groups such that the observables at one receiver station at one time epoch constitute a group.

2. For each group, the SD observables are projected to zenith and averaged to yield a pseudo-ZTD, using a predetermined mapping function.

3. The pseudo-ZTD is projected back to the actual satellite zenith angles to yield the symmetric component $\mathrm{SD}_{s}$ of each SD observable.

4. The asymmetric component $\mathrm{SD}_{a}$ is finally obtained by subtracting $\mathrm{SD}_{s}$ from $\mathrm{SD}$.

Note that the resulting $\mathrm{SD}_{a}$ is not equal to $\delta_{r}^{s}$ in (1), because the determination of $\mathrm{SD}_{a}$ does not make use of separate mapping functions for hydrostatic and wet delay components.
Furthermore, a concept of asymmetricity $r_{a}$ is introduced as

$r_{a}=\frac{\left|\mathrm{SD}_{a}\right|}{\mathrm{SD}}$

associated to an $\mathrm{SD}_{a} . \quad r_{a}$ is a measure of the azimuthally asymmetric contribution to an SD observation or to a model counterpart.

Errors of the predetermined mapping function are recognized to contribute to the calculation of $\mathrm{SD}_{a}$ even in a case of a perfectly symmetric atmosphere. Specific attention is taken in order to find the mapping function that most accurately projects the SD observables to zenith (step 2) and back to the satellite zenith angle (step 3). Performances of hydrostatic and wet mapping functions of Niell (1996) and Boehm et al. (2006) have been evaluated through histograms of $\mathrm{SD}_{a}$. In principle, the better the mapping function, the narrower the histogram, and the closer is the mean $\mathrm{SD}_{a}$ to zero. As a result, the hydrostatic mapping function of Niell (1996) is selected for this study.

\section{Asymmetricity in the SD observations}

In this Section, a statistical approach is taken in order to focus on the azimuthally asymmetric properties of the SD observations. Mean value of asymmetricity $r_{a}$ of the SD observations is 0.82 parts per thousand (ppt). Table 1 shows the numbers of the SD observations at different satellite zenith angle intervals, together with the percentages of those SD observations that exceed certain threshold values of $r_{a}$ at different zenith angle intervals. Due to observing geometry, 
Table 1. Numbers of the SD observations (\#SD) and percentages of those observations that exceed the asymmetricity thresholds $0.5,1$, 2 , and 3 parts per thousand (ppt) at different zenith angle intervals.

\begin{tabular}{cccccc}
\hline Interval & $\#$ SD & $0.5 \mathrm{ppt}$ & $1 \mathrm{ppt}$ & $2 \mathrm{ppt}$ & $3 \mathrm{ppt}$ \\
\hline $0^{\circ}-5^{\circ}$ & 3566 & 64.2 & 36.3 & 8.10 & 1.37 \\
$5^{\circ}-15^{\circ}$ & 18365 & 62.3 & 33.2 & 6.98 & 1.24 \\
$15^{\circ}-25^{\circ}$ & 25901 & 59.0 & 29.3 & 5.15 & 0.76 \\
$25^{\circ}-35^{\circ}$ & 32047 & 56.9 & 26.4 & 3.92 & 0.46 \\
$35^{\circ}-45^{\circ}$ & 36842 & 56.0 & 25.5 & 3.64 & 0.44 \\
$45^{\circ}-55^{\circ}$ & 44382 & 57.2 & 26.5 & 3.93 & 0.57 \\
$55^{\circ}-65^{\circ}$ & 51088 & 59.0 & 29.1 & 5.47 & 0.97 \\
$65^{\circ}-75^{\circ}$ & 57822 & 64.4 & 36.4 & 8.74 & 1.77 \\
$75^{\circ}-80^{\circ}$ & 26591 & 71.2 & 46.2 & 15.4 & 3.91 \\
$0^{\circ}-80^{\circ}$ & 296604 & 60.5 & 31.3 & 6.47 & 1.21 \\
\hline
\end{tabular}

the number of observations increases rather uniformly with increasing zenith angle. The first threshold, $0.5 \mathrm{ppt}$, is exceeded by $60 \%$ of the observations, and nearly one third of the observations exceed the threshold of 1 ppt. About $1 \%$ of the observations exceed the threshold of 3 ppt. These percentages depend on the satellite zenith angle interval. The observations at the largest zenith angles contain more asymmetricity than the other observations. From geometrical perspective, this is not surprising, since those observations sense atmospheric regions farther away from the receiver station than the other SD observations.

Table 1 also indicates that the observations near zenith can be notably asymmetric. This is a rather surprising result, which could perhaps be explained as follows. The geodetic network solution is mostly contributed by the measurements from large zenith angles, because more satellites are visible there than near zenith. Consequently, the fitting residuals of the network solution appear to be large at small zenith angles. This phenomenon is solely due to the observing geometry and it has no relation to the atmospheric asymmetry.

The statistical behaviour of asymmetricity is studied also at separate GPS receiver stations. It is found that there are considerable differences between different receiver stations (not shown). The differences can be related to topographical effects, such as land-sea distribution and orography, or to differences in the receiver station equipment, including receiver and antenna types. Moreover, the effect of multipath propagation is highly dependent on the surroundings of the receiver. Unfortunately, the data set used in this study does not allow to draw any more specific conclusions on the receiver station dependency.

Asymmetricity threshold of 3 ppt corresponds to an asymmetric delay component of about 7,8 and $15 \mathrm{~mm}$ at the satellite zenith angles of $0^{\circ}, 30^{\circ}$ and $60^{\circ}$, respectively. The SD model counterpart error standard deviation is of nearly equal magnitude, but the SD observation error standard deviation is roughly 1.7 times larger (Järvinen et al., 2007). Thus, SD

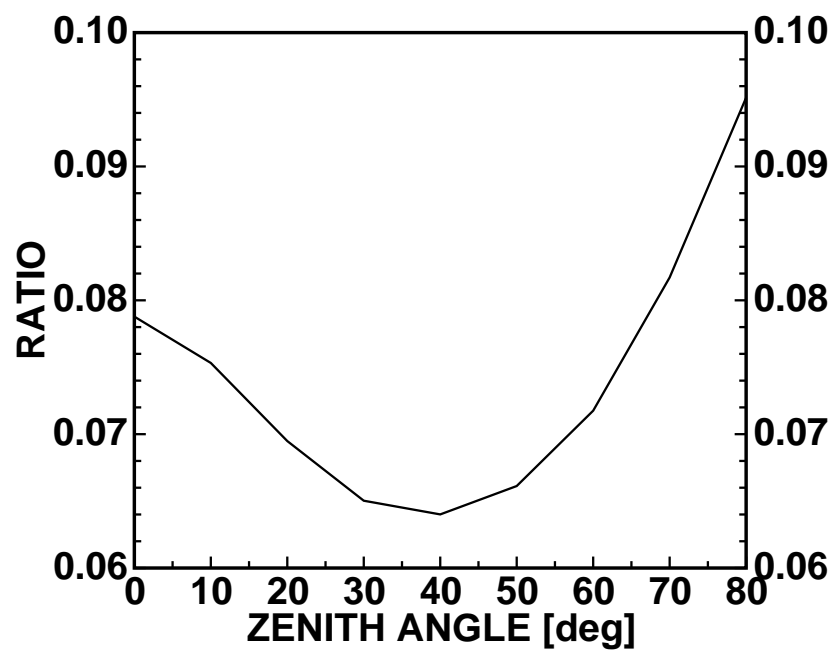

Fig. 2. Ratio of the standard deviations of the normalized asymmetric and symmetric components of SD observations as a function of satellite zenith angle.

observation error standard deviation clearly exceeds the $3 \mathrm{ppt}$ level of asymmetric delay component. Since the majority of the SD observations exhibits asymmetricity less than $3 \mathrm{ppt}$, the potential of the SD observations to NWP applications is somewhat doubtful. On the other hand, the representativeness of the SD observations will increase through increasing NWP grid resolutions in the future. This is expected to decrease the effective SD observation error from data assimilation point of view. Nevertheless, all efforts aiming at increasing the SD observation accuracy would be appreciated. These efforts might include advances, for instance, at the receiver station equipment, increase in the accuracy of satellite orbit data, improvement in the treatment of the ionospheric refraction, or other breakthroughs in the SD processing procedure.

Comparing the variabilities of the symmetric and asymmetric delay components provides another approach for assessing the asymmetric information content of the SD observations. For this purpose, standard deviations of the symmetric and asymmetric delay components are calculated at zenith angle intervals $0^{\circ}-5^{\circ}, 5^{\circ}-15^{\circ}, \ldots$, and $75^{\circ}-80^{\circ}$. The delay components are normalized by the hydrostatic mapping function of Niell (1996) prior to determination of the standard deviations. Figure 2 plots the ratio of the standard deviations of the asymmetric and symmetric delay components as a function of satellite zenith angle. The standard deviation of the asymmetric delay component appears to be between $6-10 \%$ of the standard deviation of the symmetric delay component. The shape of the curve, showing maxima at both small and large zenith angles, is similar to the columns of Table 1. Overall, it is concluded that the variability of SD is mainly due to variability of the symmetric delay component. 


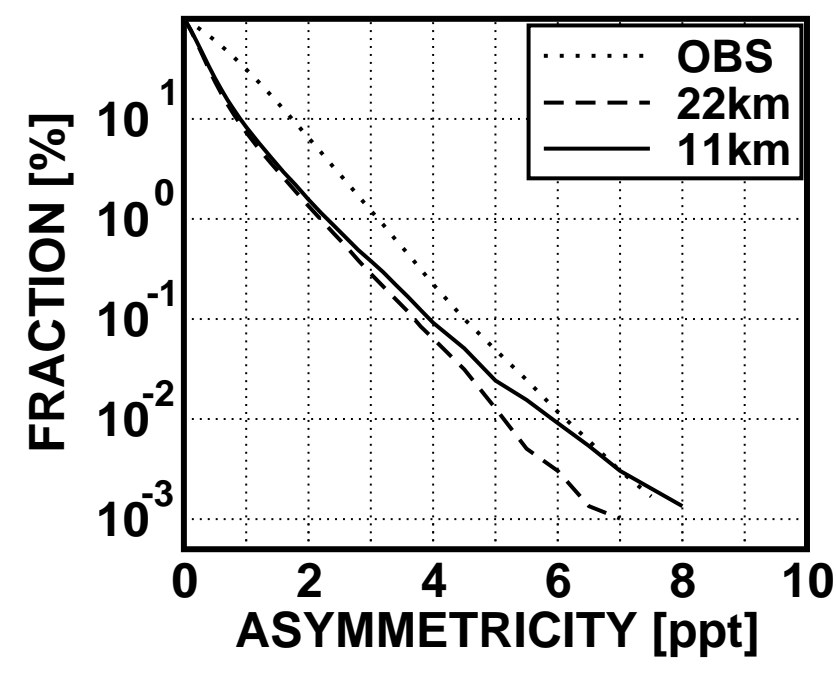

Fig. 3. Percentages of the SD observations and the model counterparts exceeding the given threshold of asymmetricity. Observations (dotted line) and model counterparts with a grid spacing of $22 \mathrm{~km}$ (dashed line) and $11 \mathrm{~km}$ (solid line).

\section{Asymmetricity in the NWP model counterparts}

In the previous section, the magnitude of the asymmetricity of the SD observations was studied in terms of percentages of observations exceeding certain threshold values. This Section provides a statistical comparison of the asymmetricity properties of the SD model counterparts with those of the SD observations. Comparison of single observations with their model counterparts will not be attempted yet. Motivation for this approach rises from properties of the analysis increments in data assimilation. The horizontal resolution of the analysis increments is governed by the so-called structure functions, which determine the spreading of information from observations to the model grid, taking multivariate balances into account (Berre, 2000). The structure functions are determined in a way, which leads to domination of synoptic scales in the analysis increments. The finer scale observational information is filtered out. The fine scale information in the analysis is provided solely by the background field and is generated by the forecast model through e.g. model physiography and land-sea distribution (Gustafsson et al., 2001).

The dotted line in Figs. 3 and 4 shows the percentages of the SD observations exceeding given thresholds. The statistics are calculated over all satellite zenith angles. Also the corresponding curves for the model counterparts with the four different horizontal grid resolutions are plotted. Figure 3 shows the curves for the model run with $22 \mathrm{~km}$ (dashed line) and $11 \mathrm{~km}$ (solid line) grid spacings, and Fig. 4 shows the curves for $5.6 \mathrm{~km}$ (dashed line) and $2.8 \mathrm{~km}$ (solid line) grid spacings.

Among the large group of the most symmetric SD observations, covering $99 \%$ of all cases, the observed asymmetricity

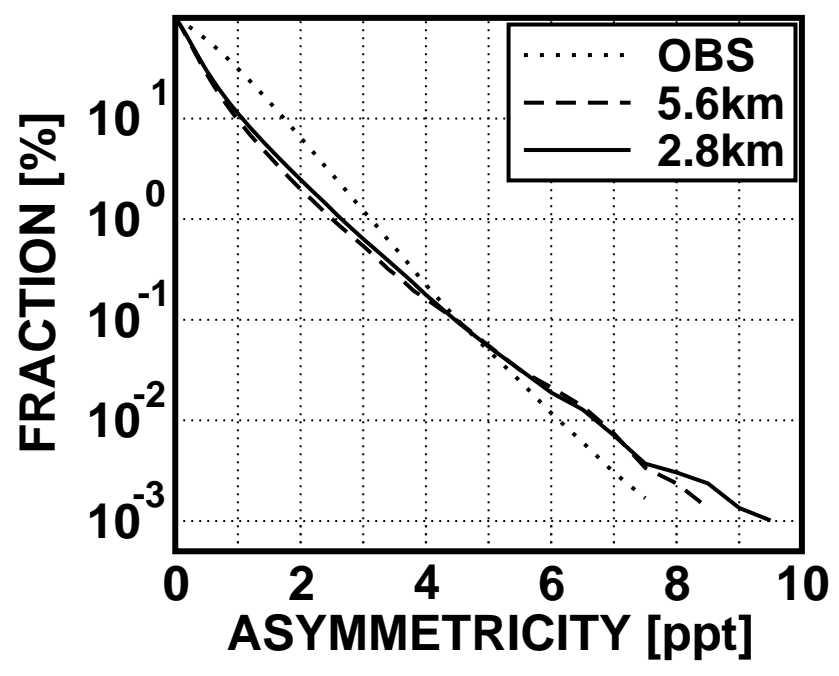

Fig. 4. As Fig. 3, but for grid spacings of $5.6 \mathrm{~km}$ (dashed line) and $2.8 \mathrm{~km}$ (solid line).

is below 3 ppt. The model forecasts, especially those made with grid spacings 22 and $11 \mathrm{~km}$ (Fig. 3), fail to represent the asymmetricity to a similar extent in these cases and only reach 2 ppt. This could be explained by the SD observations containing a significant amount of non-meteorological measurement noise, resulting in too large asymmetricity in symmetric atmospheric conditions. Another possible explanation is that the currently used NWP systems are unable to simulate these small scale features. The fact, that an increase in the horizontal resolution results in closer agreement with the observations (Fig. 4), supports the latter interpretation. However, without additional data it is not possible to exclude the former interpretation.

For asymmetricities above $3 \mathrm{ppt}$, the percentage curves of the model forecasts tend to approach the observation curve. At asymmetricity values higher than $5 \mathrm{ppt}$, the model counterparts with a 5.6 and $2.8 \mathrm{~km}$ grid spacing show even more asymmetricity than revealed by the observations. It is interpreted that part of the asymmetric information is lost in the $\mathrm{SD}$ processing procedure in cases of extreme atmospheric asymmetricity. This interpretation is in line with the simulation study reported by Elosegui and Davis (2004), and it holds for $0.05 \%$ of the observations in the present data set.

Moreover, since the curves for the model counterparts with a 5.6 and $2.8 \mathrm{~km}$ grid spacing are very close to each other, it is concluded that the NWP grid with horizontal spacing of $5.6 \mathrm{~km}$ is likely to be dense enough in order to model the azimuthal asymmetricity of the SD observations in the present data set with a reasonable accuracy. This means that data assimilation of the SD observations can be expected to be beneficial compared to data assimilation of the ZTD observations in NWP systems with horizontal grid spacing of around $5 \mathrm{~km}$ or less. 

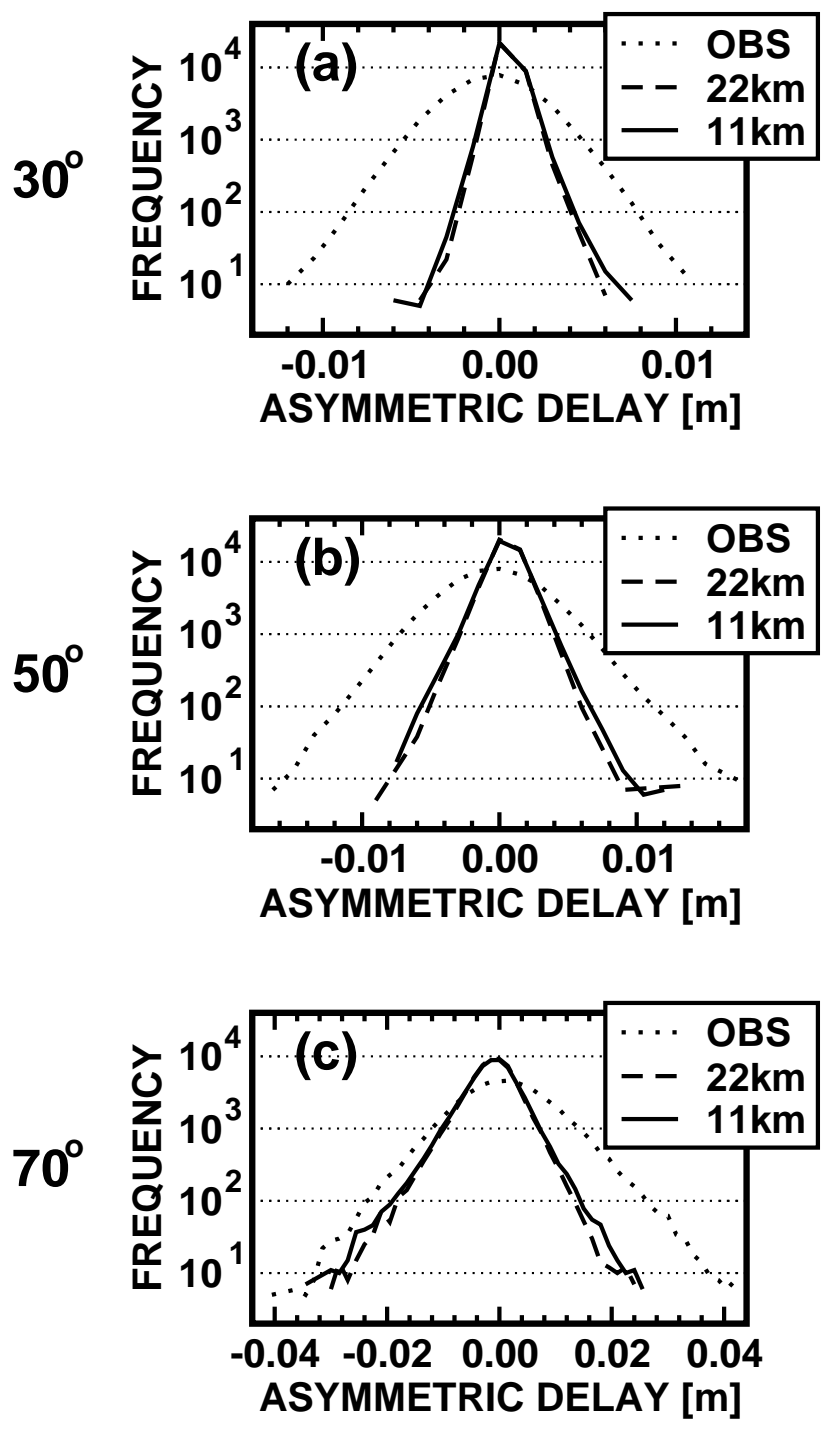

Fig. 5. Frequency distributions of the asymmetric delay components at zenith angle intervals $30^{\circ} \pm 5^{\circ}$ (a), $50^{\circ} \pm 5^{\circ}$ (b) and $70^{\circ} \pm 5^{\circ}$ (c). Observations (dotted line) and model counterparts with a grid spacing of $22 \mathrm{~km}$ (dashed line) and $11 \mathrm{~km}$ (solid line).

Figures 5 and 6 show the frequency distributions of $\mathrm{SD}_{a}$ at three satellite zenith angle intervals. Figure 5 shows the distributions of the SD observations (dotted line) and the model counterparts with a $22 \mathrm{~km}$ (dashed line) and $11 \mathrm{~km}$ (solid line) grid spacing, and Fig. 6 shows the distributions of the model counterparts with a $5.6 \mathrm{~km}$ (dashed line) and $2.8 \mathrm{~km}$ (solid line) grid spacing. At all zenith angle intervals, the distributions of the model counterparts are too narrow compared with the observed distributions. However, increasing the NWP model's horizontal resolution generally increases the spread towards the observed distribution. At the zenith angle interval of $70^{\circ} \pm 5^{\circ}$, the distributions of the model counterparts at resolutions of $5.6 \mathrm{~km}$ and $2.8 \mathrm{~km}$ (panel (c) of Fig. 6) are very similar. On the other hand, the $2.8 \mathrm{~km}$ reso-
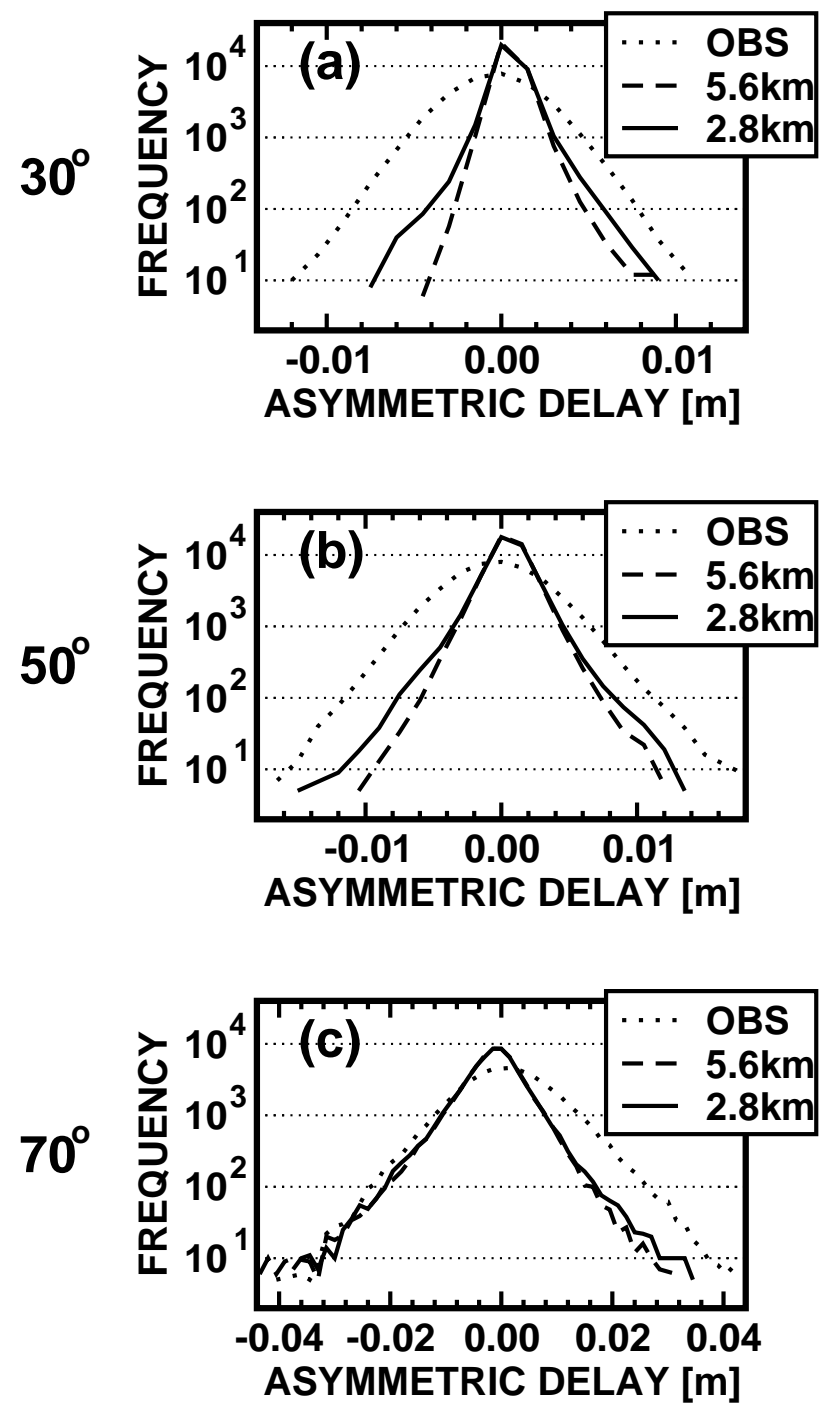

Fig. 6. As Fig. 5, but for grid spacings of $5.6 \mathrm{~km}$ (dashed line) and $2.8 \mathrm{~km}$ (solid line).

lution provides clearly the best agreement with observations at the smaller zenith angles (panels (a) and (b) in Figs. 5 and 6). In conclusion, $5.6 \mathrm{~km}$ grid spacing appears to be sufficient for explicit modelling of the asymmetricities in the SD observations at the largest zenith angles, where the observed azimuthal asymmetricity is relatively large (see Table 1). Decreasing the grid spacing closer to $2.8 \mathrm{~km}$ is probably necessary in order to make the best use of the observations at zenith angles smaller than $65^{\circ}$.

The frequency distributions of the model counterparts are generally not symmetric around zero. To be more specific, the distributions are skewed towards positive asymmetric delays at small zenith angles and towards negative asymmetric delays at large zenith angles. This behaviour can be seen also in frequency distributions corresponding to separate receiver stations (not shown). Such a behaviour suggests that 
the applied mapping function is inconsistent with the zenith angle dependency of the SD model counterparts. This is in line with the zenith angle dependent bias in the observation minus model background statistics reported earlier by Eresmaa and Järvinen (2006). This study applies no algorithm for bias correction. Note that it is impossible to say whether the observations or the model counterparts are more responsible for the bias. The symmetric distributions of SD observations in Figs. 5 and 6 most likely follow from the fact that the SD observation processing has made use of the same mapping function as the one applied in this study.

It is important to note that the asymmetric delay components of the model counterparts do not follow gaussian distributions, while those of the SD observations do. It seems that the model counterparts contain too little asymmetricity in the relatively symmetric cases, but overestimate the asymmetricity in the cases of extreme asymmetricity. The reason for this behaviour is unknown at the moment.

\section{Intercomparison in highly asymmetric cases}

In this section, the comparison is extended to pairs of observations and their model counterparts, focusing on cases of exceptionally high asymmetricity as revealed by either observations or their model counterparts.

The asymmetricity $r_{a}$ measures the azimuthally asymmetric contribution to an SD observation or to a model counterpart. Even though the high values of observed $r_{a}$ can be attributed to atmospheric properties in the vicinity of the GPS receiver station, it is not obvious that all such cases are meteorologically interesting. This is due to a number of uncertainties affecting the microwave propagation, signal reception and GPS data processing. In this Section, the NWP model forecasts are considered as reference atmospheres, which either do or do not support the interpretation of atmospheric properties as the source of high observed asymmetricity.

\subsection{Support from the NWP model forecasts}

In order to investigate whether the observed high asymmetricity values are signatures of atmospheric properties, the following procedure is applied:

1. The SD observations are ordered according to increasing $r_{a}$. The observations exceeding the threshold $r_{a}$ value of $3.12 \mathrm{ppt}$ are considered highly asymmetric. The threshold is chosen such that the highly asymmetric observations cover $1 \%$ of all SD observations.

2. The model counterparts are ordered in a similar manner as the observations. The threshold value corresponding to $1 \%$ of the model counterparts varies between 2.19 and $2.65 \mathrm{ppt}$, depending on the horizontal grid spacing.
Table 2. Percentages, and their $95 \%$ and $99 \%$ confidence limits, of highly asymmetric observations that are interpreted to indicate real atmospheric asymmetry at different numbers of supporting model forecasts (SMF).

\begin{tabular}{cccc}
\hline SMF & Observations & $95 \%$ & $99 \%$ \\
\hline$\geq 1$ & 20.1 & 17.4 & 18.0 \\
$\geq 2$ & 8.73 & 7.49 & 7.86 \\
$\geq 3$ & 4.55 & 3.81 & 4.28 \\
4 & 2.43 & 2.06 & 2.12 \\
\hline
\end{tabular}

3. The counterparts to the highly asymmetric observations, detected by the receiver station identification, observing time and the Satellite Vehicle Number (SVN), are searched one by one from the group of highly asymmetric model counterparts. Time difference of up to three hours is allowed between the observation and the model background.

4. If there is a matching highly asymmetric model counterpart to the highly asymmetric observation, the NWP model is concluded to support the interpretation that this observation indicates a real atmospheric asymmetry.

5. The steps $2-4$ are repeated four times corresponding to the model counterpart data sets at four different NWP grid resolutions.

The interpretation of a highly asymmetric SD observation showing real atmospheric asymmetry is thus supported by up to four NWP model forecasts. The larger the number of supporting model forecasts (SMF) is, the more convincing is the interpretation. The second column of Table 2 shows the percentages of the SD observations receiving support of at least one, two, three or four SMF. A fraction of the highly asymmetric SD observations can be concluded to indicate a real atmospheric asymmetry. This result shows the balance between the noise influencing the SD processing and the meteorological, azimuthally asymmetric, information contained in the SD observations.

\subsection{Statistical significance of the support}

It is obvious that the procedure described above would result in non-zero percentages in Table 2 even in a case of artificial SD observations being definitely independent of real atmospheric conditions. In other words, some unknown percentage of the artificial observations would accidentally receive support from the NWP model forecasts and would further be concluded to show an asymmetric meteorological signal. Therefore, confidence limits to the percentages in Table 2 are estimated in the following way: A sample of one hundred sets of gaussian random numbers is constructed. Each set consists of 296604 values being definitely not related to real 
atmosphere. Each individual random number in each set is attached to one SD observation in the original data set; each random number is thus considered to represent asymmetricity of a single SD observation. The procedure applied above to the data set of observed asymmetricities is then applied one by one to each of the sets of random numbers. Repeating the procedure over the sample of one hundred sets allows to assign the confidence limits. Note that the statistical parameters of the random numbers are irrelevant for this study. The conclusions of the experiment will be sensitive only to the order at which the random numbers happen to occur.

The resulting $95 \%$ and $99 \%$ confidence limits are included in the third and fourth columns of Table 2 for each level of SMF. The percentage of highly asymmetric SD observations indicating real atmospheric asymmetry exceeds the $99 \%$ confidence limit at all SMF levels. The subsequent conclusion is that the SD observations contain a statistically significant asymmetric meteorological signal.

\section{Conclusions}

This article introduces and applies the concept of asymmetricity on the SD observations and their NWP model counterparts. Asymmetricity expresses the contribution of azimuthal asymmetry to the total SD. On the basis of the results presented in the previous Sections, the following answers are provided to the questions listed in Sect. 1:

- How large is the contribution of the azimuthally asymmetric information to the SD observations? The asymmetric contribution, i.e. asymmetricity, is of the order of a few parts per thousand of the absolute delay value. In the extreme cases, mainly at satellite zenith angles larger than $65^{\circ}$, the asymmetricity can exceed the threshold of 5 ppt. It is exceptional that the asymmetric contribution is larger than the assumed standard deviation of the SD observation error.

- Is the azimuthal asymmetry in the SD observations related to real atmospheric asymmetry? As far as the NWP model represents the true atmosphere, it seems to be related. This conclusion holds for the cases of extreme asymmetricity and it is statistically significant at the confidence level of $99 \%$.

- Are the currently operational NWP systems, with horizontal grid spacings of around $10-20 \mathrm{~km}$, able to represent the scales appropriate for extraction of the asymmetric information? The HIRLAM NWP system is only partially able to represent the asymmetric properties of the SD observations. The closest agreement with the observations is obtained at zenith angles larger than $65^{\circ}$.

- Can the NWP model's representation of the azimuthal asymmetry be improved by increasing the horizontal resolution? Yes, it can be improved. If the SD observations at zenith angles larger than $65^{\circ}$ are considered, the horizontal grid spacing of around $5 \mathrm{~km}$ seems to be dense enough in order to explicitly model the asymmetricity. However, modelling of the asymmetry at smaller zenith angles requires a denser grid.

This study makes use of a hydrostatic limited area NWP system, originally intended to provide synoptic scale guidance. It is possible that the conclusions would be somewhat different if a finer scale non-hydrostatic NWP system was used. Moreover, the results are expected to depend also on the horizontal resolution of the observing systems assimilated in the model.

The currently operational limited area NWP systems are already close to the highest horizontal resolution used in this study. Therefore, the NWP data assimilation is expected to be able to make use of the whole information content of the SD observations in near future. At the moment, the most obvious obstacle for SD data assimilation is the lack of data processing in near real time.

Acknowledgements. The funding from the EU FP5 project "Targeting Optimal Use of GPS Humidity Measurements in Meteorology" (TOUGH) in 2003-2006 and from the TEKES project "Geophysically Assisted Satellite Positioning" in 2004-2006 is thankfully acknowledged. TOUGH is a shared-cost project (contract EVG1-CT-2002-00080) co-funded by the Research DG of the European Commission within the RTD activities of the Environment and Sustainable Development sub-programme (5th Framework Programme). TEKES is the Finnish Funding Agency for Technology and Innovation. The authors are grateful for Hans van der Marel (Technical University of Delft, the Netherlands) for providing the SD observations.

Edited by: K. Hamilton

\section{References}

Berre, L.: Estimation of synoptic and mesoscale forecast error covariances in a limited area model, Mon. Wea. Rev., 128, 644667, 2000.

Bevis, M., Businger, S., Herring, T., Rocken, C., Anthes, R., and Ware, R.: GPS meteorology: Remote sensing of atmospheric water vapor using the Global Positioning System, J. Geophys. Res., 97, 15 787-15 801, 1992.

Boehm, J., Niell, A., Tregoning, P., and Schuh, H.: Global Mapping Function (GMF): A new empirical mapping function based on numerical weather model data, Geophys. Res. Lett., 33, L07304, doi:10.1029/2005GL025546, 2006.

Bouttier, F.: The AROME mesoscale project, in: Proceedings of a seminar on Recent developments in data assimilation for atmosphere and ocean, 8-12 September 2003, European Centre for Medium-Range Weather Forecasts, Shinfield Park, Reading, Berkshire, England, pp. 433-448, 2004.

de Haan, S., van der Marel, H., and Barlag, S.: Comparison of GPS slant delay measurements to a numerical model: case study of a cold front passage, Phys. Chem. Earth, 27, 317-322, 2002. 
De Pondeca, M. and Zou, X.: A case study of the variational assimilation of GPS zenith delay observations into a mesoscale model, J. Appl. Meteorol., 40, 1559-1576, 2001.

Elgered, G., Plag, H.-P., van der Marel, H., Barlag, S., and Nash, J., eds.: COST Action 716: Exploitation of ground-based GPS for operational numerical weather prediction and climate applications, Final report, Rep. EUR 21639, European Union, 234 pp., 2005.

Elosegui, P. and Davis, J.: Accuracy assessment of GPS slant-path determinations, in: Proc. International Workshop on GPS meteorology, Tsukuba, Japan, 14-17 Jan 2003, edited by: Iwabuchi, T. and Shoji, Y., 1-35-1-1-35-6, 2004.

Eresmaa, R. and Järvinen, H.: An observation operator for groundbased GPS slant delays, Tellus, 58A, 131-140, 2006.

Gustafsson, N., Berre, L., Hörnquist, S., Huang, X.-Y., Lindskog, M., Navascués, B., Mogensen, K. S., and Thorsteinsson, S.: Three-dimensional variational data assimilation for a limited area model. Part I: General formulation and the background error constraint, Tellus, 53A, 425-446, 2001.

Ha, S.-Y., Kuo, Y.-H., Guo, Y.-R., and Lim, G.-H.: Variational assimilation of slant path wet delay measurements from a hypothetical ground-based GPS network. Part I: Comparison with precipitable water assimilation, Mon. Wea. Rev., 131, 2635-2655, 2003.

Hollingsworth, A., Viterbo, P., and Simmons, A. J.: The relevance of numerical weather prediction for forecasting natural hazards and for monitoring the global environment, Tech. Memo. 361, ECMWF, European Centre for Medium-Range Weather Forecasts, Shinfield Park, Reading, Berkshire, England, 29 pp., 2002.

Järvinen, H., Eresmaa, R., Vedel, H., Salonen, K., Niemelä, S., and de Vries, J.: A variational data assimilation system for groundbased GPS slant delays, Q. J. Roy. Meteor. Soc., in press, 2007.
Lindskog, M., Gustafsson, N., Navascués, B., Mogensen, K. S., Huang, X.-Y., Yang, X., Andræ, U., Berre, L., Thorsteinsson, S., and Rantakokko, J.: Three-dimensional variational data assimilation for a limited area model. Part II: Observation handling and assimilation experiments, Tellus, 53A, 447-468, 2001.

Liu, H. and Xue, M.: Retrieval of moisture from slant-path water vapor observations of a hypothetical GPS network using a threedimensional variational scheme with anisotropic background error, Mon. Wea. Rev., 134, 933-949, 2006.

MacDonald, A. E., Xie, Y., and Ware, R. H.: Diagnosis of threedimensional water vapor using a GPS network, Mon. Wea. Rev., 130, 386-397, 2002.

Niell, A.: Global mapping functions for the atmosphere delay at radio wavelengths, J. Geophys. Res., 101, 3227-3246, 1996.

Niemelä, S. and Fortelius, C.: Applicability of large scale convection and condensation parameterization to meso- $\gamma$-scale HIRLAM: A case study of a convective event, Mon. Wea. Rev., 133, 2422-2435, 2005.

Undén, P., Rontu, L., Järvinen, H., Lynch, P., Calvo, J., Cats, G., Cuxart, J., Eerola, K., Fortelius, C., Garcia-Moya, J. A., Jones, C., Lenderlink, G., McDonald, A., McGrath, R., Navascués, B., Woetman Nielsen, N., Ødegaard, V., Rodriguez, E., Rummukainen, M., Rõõm, R., Sattler, K., Hansen Sass, B., Savijärvi, H., Wichers Schreur, B., Sigg, R., The, H., and Tijm, A.: HIRLAM-5 Scientific Documentation, Hirlam-5 Project, available from Hirlam-5 Project, c/o Per Undén, SMHI, S-60176, Norrköping, Sweden. 144 pp., 2002.

Vedel, H. and Huang, X.-Y.: Impact of Ground Based GPS Data on Numerical Weather Prediction, J. Meteor. Soc. Japan, 82, 459472, 2004. 